\title{
Pflanzen in Schau- und Lehrgärten am Beispiel von Hans Felix Kammeyer und Des Kammeyergartens in Dresden-Pillnitz
}

\author{
Anke Mühlpfordt
}

$\mathrm{P}$ illnitz besitzt als Zentrum der gärtnerischen Lehre und Forschung in Sachsen eine lange, deutschlandweite Tradition. Über viele Jahrzehnte wurden hier national und international anerkannte Gartenbauinspektoren und -ingenieure sowie Gartenbaumeister und -techniker ausgebildet. Heute bestehen an diesem Standort renommierte Forschungs- und Lehrinstitutionen, wie das Sächsische Landesamt für Umwelt, Landwirtschaft und Geologie, das Julius-Kühn-Institut (Institut für Züchtungsforschung) sowie die Hochschule für Technik und Wirtschaft (HTW), Fakultät Landbau/Umwelt/Chemie (früher Landbau und Landespflege).

Der nach und zu Ehren von Hans Felix Kammeyer benannte Kammeyergarten ist Bestandteil der Außenanlagen der Fakultät. Der Campus in Pillnitz befindet sich nordöstlich des Pillnitzer Schlosses und erstreckt sich beidseitig des Pillnitzer Platzes. Der Kammeyergarten umfasst das Gebiet des ehemaligen Schau- und Versuchsgartens sowie des damaligen Bienengartens. Er wird vom Schindler- und Mitschurinbau sowie der großen Schlossscheune und der Schlossgartenmauer begrenzt.

Der Pillnitzer Platz mit den der HTW zugehörigen Freianlagen ist als Kulturdenkmal und als Bestandteil des Denkmalschutzgebietes »Elbhänge« gemäß $\$ \$ 2$ und 21 Sächsisches Denkmalschutzgesetz (SächsDSchG) geschützt.

Anlass für die Beschäftigung mit der Geschichte und Entwicklung der Lehr- und Schauanlagen am Pillnitzer Platz war die durch das Büro May Landschaftsarchitekten
Dresden zwischen 2012 und 2013 erarbeitete Denkmalpflegerische Rahmenkonzeption für den Kammeyergarten und das sogenannte Lindendreieck.

Der Kammeyergarten ist, obgleich nicht mehr vollständig erhalten, ein wichtiges Beispiel für das Wirken und Schaffen von Hans Felix Kammeyer (1893-1973), einem bedeutenden Dresdner Gartenarchitekten, Pädagogen und Dendrologen. An der Lehranstalt war er in den 1920er und später in den 1950er Jahren maßgeblich an der Gestaltung und Weiterentwicklung der Lehrund Schaugärten in Pillnitz beteiligt. Kammeyer zeichnete sich zudem durch eine umfangreiche publizistische Tätigkeit in den Bereichen Gartenarchitektur, Geschichte der Gartenkunst und Gartendenkmalpflege, Dendrologie, Heimatschutz, Ingenieurbiologie sowie praktisches Gartenwissen aus.

Hans Felix Kammeyer (Abb. 1) wurde 1893 in Berlin-Charlottenburg geboren. Seine gärtnerische Ausbildung absolvierte er von 1912 bis 1914 an der Höheren Gärtnerlehranstalt Berlin-Dahlem. Nachfolgend war Kammeyer als Gärtnergehilfe in verschiedenen Gärtnereien und Baumschulen, unter anderem der Baumschule Lorberg bei Berlin, tätig. Zwischen 1916 und 1920 erfolgte die Ausbildung zum Gartenbautechniker an der Höheren Gärtnerlehranstalt in Proskau. Anschließend arbeitete er als freischaffender Gartenarchitekt vorrangig in Mecklenburg und Pommern. Seine Zweite Staatsprüfung zum »Staatlich diplomierten Gartenbauinspektor « legte er 1925 an der Höheren Gärtnerlehranstalt Berlin-Dahlem ab. ${ }^{1}$ 


\section{Einrichtung der Höheren Staatslehr- anstalt in Pillnitz}

Nach dem Ersten Weltkrieg wurde Pillnitz als zukünftiger Standort für eine gartenbauliche Lehranstalt bestimmt. Die überaus günstigen klimatischen Verhältnisse und die unmittelbare Nähe zum Schlosspark Pillnitz sowie der Bestand an renommierten gärtnerischen Einrichtungen - neben der 1913 nach Pillnitz verlagerten Königlichen Hofgärtnerei an der Lohmener Straße (ab 1920 Staatliche Versuchs- und Beispielgärtnerei) das Staatliche Kammergut und das Obstgut der Stadt Dresden in Hosterwitz - gaben wohl die Veranlassung dazu.

Die »Höhere Staatslehranstalt für Gartenbau« wurde am 1. Mai 1922 in Pillnitz gegründet. Darin ging die seit Ende des 19. Jahrhunderts in Dresden existierende Gartenbauschule des Gartenbauverbandes mit auf. Aufgrund der Verstaatlichung der königlichen Besitzungen nach dem Ersten Weltkrieg boten sich Räumlichkeiten für die Lehranstalt in den beiden ehemaligen Marstallgebäuden des Pillnitzer Schlosses an, die daraufhin entsprechend ausgebaut wurden.

Die Staatslehranstalt diente der beruflichen Ausbildung von Gartenbautechnikern (Erste Staatsprüfung) und diplomierten Gartenbauinspektoren (Zweite Staatsprüfung), der Weiterbildung von Gartenbautreibenden sowie der allgemeinen Förderung des Gartenbaus.

\section{Der Lehrgarten der 1920er Jahre}

Im September 1922 wurde Kammeyer als Dozent für Gartengestaltung, Garten- und Baukunst an die neu gegründete Höhere Staatslehranstalt für Gartenbau in Pillnitz berufen. Die Betreuung der Stauden- und Gehölzsammlung sowie die Ausgestaltung der Freiflächen an der Lehranstalt waren ein weiterer wichtiger Bestandteil seiner Arbeit. Diese Aufgabe übernahm er zusammen mit seinem Kollegen und Vorsteher der Abteilung Gartenkunst, dem Gartenbauinspektor
Louis Kniese (1875-1947). ${ }^{2}$ Unter der Federführung von Kammeyer sollte auf dem Gelände der Lehranstalt ein Lehr- und Versuchsgarten entstehen. Zweck und Funktion eines Lehrgartens werden seitens der Lehranstalt wie folgt dargelegt:

»Die Anschauungsmöglichkeiten unmittelbar an der Lehranstalt können durch nichts ersetzt werden, da nur sie dem Studierenden die Möglichkeit einer dauernden Beobachtung geben. Auf einem beschränkten Gelände, und mit den für solche Zwecke nur gering vorhandenen Mitteln lassen sich in den Lehrgärten weniger Beispiele der Gartengestaltung zeigen diese werden besser auf häufigen Studienfahrten in der Praxis besichtigt - als Pflanzen, Zusammenstellungen, Versuche und Beispiele ihrer Verwendung und andere Einzelheiten aus der Gartengestaltung. An der Staatslehranstalt dienen die Lehrgärten [...] gleichzeitig als Lehrmittel für den Freilandblumenbau und die Gehölzkunde, als auch den Erwerbsgartenbau. $\ll^{3}$

Der Lehrgarten der 1920er Jahre (Abb. 2) bestand aus einem Alpinum, einem biologischen Teil, einem Teichstück (Veilchenteich) sowie einem Gartenkunstteil mit einem von Kammeyer angelegten Stauden- und Blumengarten. Dieser »soll einen mit den Mitteln der Gartenkunst verschönerten Teil des Lehrgartens bilden, gleichzeitig aber auch den Anstaltsbesuchern Gelegenheit geben, Stauden und Sommerblumen und andere Schmuckpflanzen kennen zu lernen, und einen Teil des Werkstoffs für die Übungen der Bindereien liefern. «4

Der Stauden- und Blumengarten, eine gestalterisch einfache formale Anlage, war durch eine Längs- und Querachse gegliedert und bestand aus einem höher und einem tiefer gelegenen Teil. Der Geländeunterschied von etwa $50 \mathrm{~cm}$ wurde durch eine Trockenmauer abgefangen. Der höher gelegene Abschnitt des Blumengartens gliederte sich in drei quadratische Beete. Das mittlere 
Wechselflorbeet wurde im Frühjahr und Herbst teppichartig mit verschiedenen Einjährigen bepflanzt. Die Mitte des Beetes war erhöht und anfangs mit einer Säule für eine Sonnenuhr besetzt. Später stand an dieser Stelle eine Putte (Abb. 3). Beidseitig des Wechselflorbeetes lagen, durch Rasenstreifen getrennt, zwei Staudenbeete. Oberhalb befand sich ein mit hohen Herbstblühern bepflanztes Beet, darin eine von einer niedrigen Hecke aus Heckenmyrte (Lonicera nitida) eingefasste Steinbank, die gleichzeitig den Endpunkt der Längsachse bildete. Westlich des Staudenbeetes führte eine kleine Treppe zum Veilchenteich.
Auf den Beeten im Blumen- und Staudengarten wurden Pflanzengesellschaften, wie zum Beispiel trockenverträgliche oder feuchtigkeitsliebende Pflanzen sowie mehr oder weniger umfangreiche Pflanzensortimente, "zum Vergleich untereinander und um ihren Wert für die Gartengestaltung zu prüfen $\aleph^{5}$ präsentiert.

Diese setzten sich aus Einjährigen, Stauden (unter anderem Hauswurz), Rosen (Buschrosen) sowie Sträuchern (unter anderem Zier-Johannisbeere) zusammen. Des Weiteren gab es eine kleine Sammlung seltener Sträucher am Veilchenteich, die als Ergänzung der Sammlung im Schlossgarten

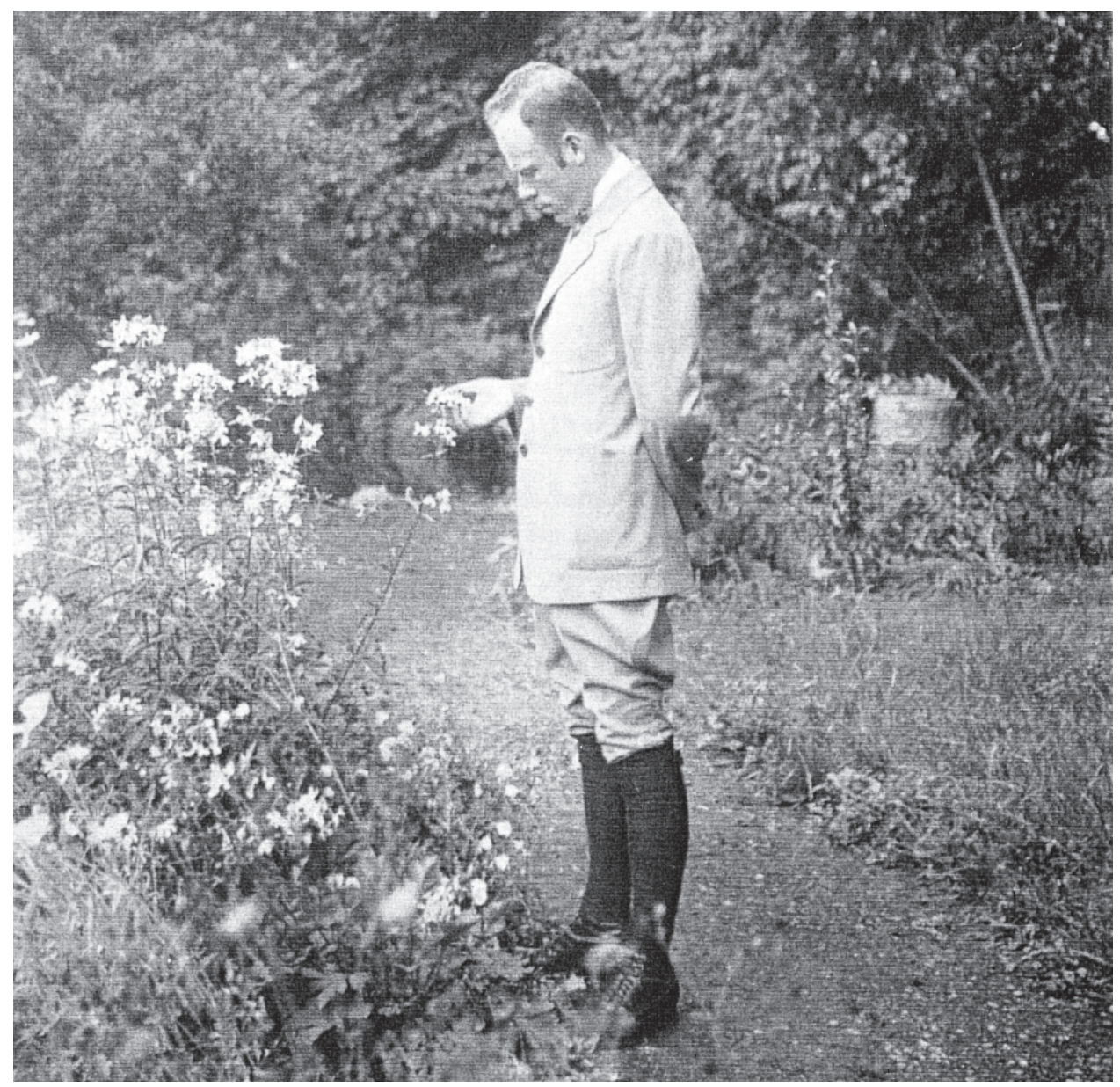

1 Hans Felix Kammeyer, 1920er Jahre (HTW Dresden Pillnitz, Nachlass Kammeyer, Bildarchiv - ohne Kennzeichnung). 
gedacht war. Auch wurden weitere Staudensortimente, wie Phlox, Astern, Primeln sowie Dahlien kultiviert. ${ }^{6}$

Kammeyer strebte in seinem Staudengarten die teppichartige Massenwirkung der Pflanzen an:

»Der Begriff Staudengarten ist uns meist schon geläufig. Was davon existiert, wirkt meist in seinem bunten Durcheinander nichtssagend, da jede Wirkung verloren geht durch die ungeheure Menge des Materials. [...] Es gibt nur eine Art von Staudengärten, die ihre Wirkung hat, das ist dort, wo schon immer 20 bis 30 Pflanzen einer Sorte vereinigt sind, die dann nur Massenwirkungen hervorbringen ${ }^{7}{ }^{7}$

Im Jahr 1926 rief man in Pillnitz eine Imkerlehrstätte ins Leben. Östlich des Veilchenteichs, an der großen Scheune, wurde auf einer $350 \mathrm{~m}^{2}$ großen Fläche ein Lehrbienenstand geschaffen. Bis 1934 erfolgte die Betreuung und Verwaltung der Imkerlehrstätte noch durch die Versuchs- und Forschungsanstalt. Später wurde ein geprüfter Bienenmeister und Berufsimker verpflichtet. $^{8}$

\section{Zeit des Nationalsozialismus bis 1945}

Zwischen 1933 und 1934 schieden aufgrund des von den Nationalsozialisten erlassenen Gesetzes zur Wiederherstellung des Berufsbeamtentums mehrere Lehrer aus dem Lehrkörper aus, darunter auch Kammeyer. In der Folgezeit war er freischaffend als Gartenarchitekt vorrangig in und um Dresden tätig und verwirklichte verschiedene Projekte, unter anderem den Villengarten am Sonnenhang in Dresden-Loschwitz. Die Gestaltung der Schmuckanlagen an den Anstaltsgebäuden erfuhr bis 1936 kaum eine Veränderung. Mit Ausbruch des Krieges rückten für die Anstalt Aufgaben auf dem Gebiet der Ernährung und der Versorgung der Bevölkerung mit Obst und Gemüse in den Vordergrund. In den letzten Kriegsjahren diente der Lehrgarten zum großen Teil als Kleingartenland und wurde auch von einer während des Krieges eingerichteten Heereslehrküche genutzt. Im Jahr 1942 musste die Anstalt schließlich geschlossen und die Lehre ausgesetzt werden.

Kurz vor Kriegsende wurde Pillnitz Ziel eines Luftangriffs, der verheerende Schäden an den Gebäuden sowie auch in den gärtnerischen Anlagen anrichtete.

\section{Der Blumengarten im Lehrgarten der Jöh. Staatslehranstalt für Gartenbau}

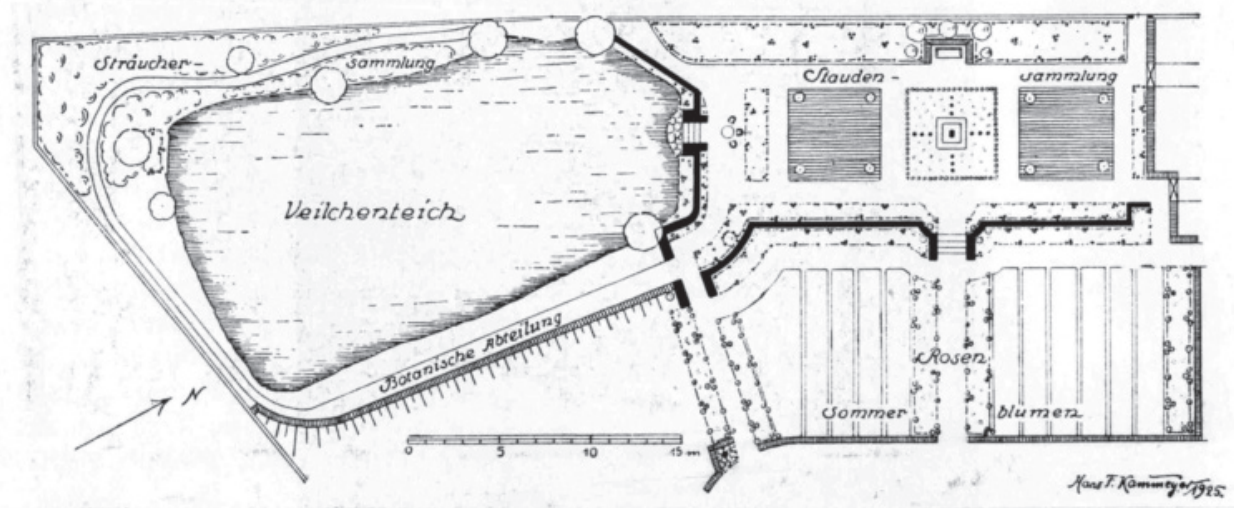

2 | Plan des Blumengartens im Lehrgarten der Höheren Staatslehranstalt für Gartenbauvon 1925 (HTW Dresden Pillnitz, Nachlass Kammeyer, „Verzeichnis meiner schriftlichen Arbeiten Hans F. Kammeyer 1911-1973«). 


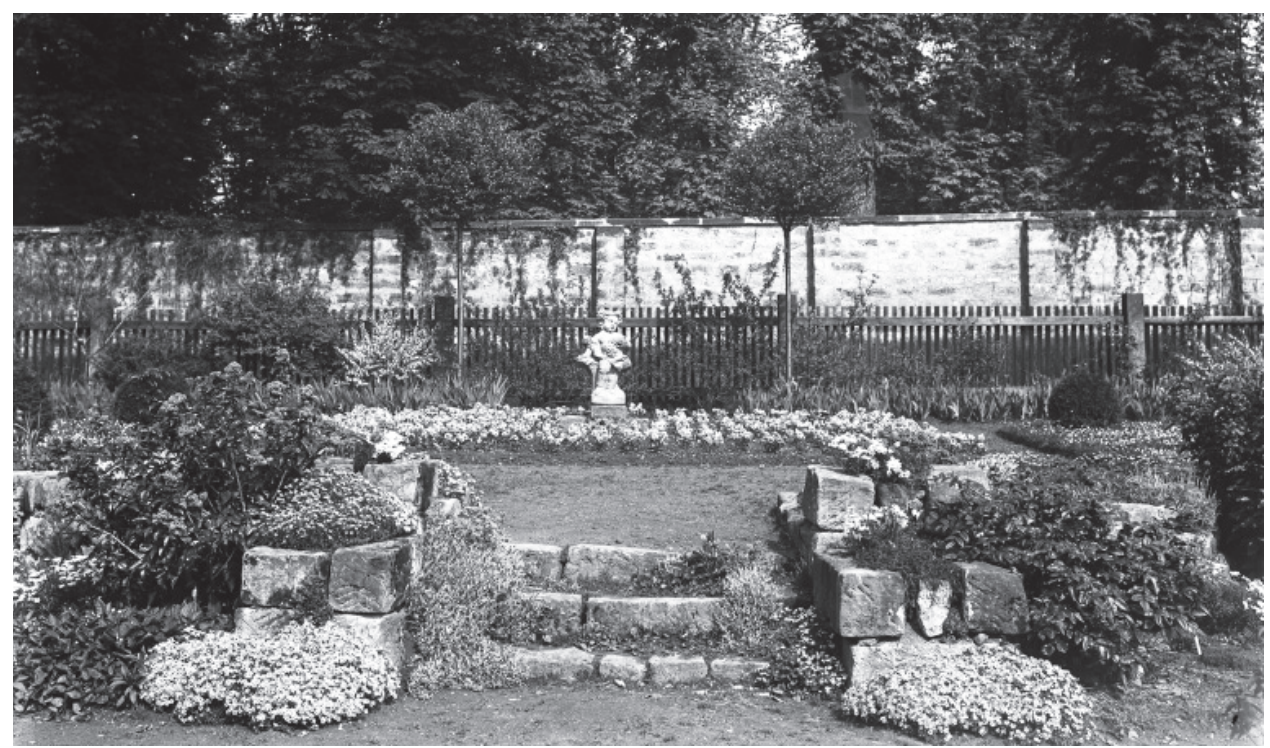

3 | Blick von Osten auf den Blumengarten, 1920er Jahre (HTW Dresden Pillnitz, Nachlass Kammeyer, KAM K57).

\section{Wiederaufnahme der Lehre 1946 und Neugestaltung des Lehrgartens und der institutseigenen Zieranlagen ab den 1950er Jahren}

Auch nach Ende des Krieges wurde ein Großteil der Fläche des ehemaligen Lehrgartens von Kleingartenpächtern, meist Betriebsangehörige der Lehranstalt, als Gartenland bewirtschaftet. Die Produktion von Obst- und Gemüse dauerte in einigen Anlagenteilen noch bis Anfang der 1950er Jahre fort.

Der Wiederaufbau der Lehranstalt erfolgte unmittelbar nach Kriegsende. Innerhalb des Lehrkörpers fand eine umfassende Reorganisation statt, ehemalige Mitglieder der NSDAP schieden aus dem Kollegium aus. Kammeyer stellte sich der Lehranstalt wieder zur Verfügung und wurde zum Direktor der Abteilung Garten- und Landschaftsgestaltung an der neu gegründeten Versuchs- und Forschungsanstalt in Pillnitz berufen.

Für die Wiederherstellung des Lehrgartens, an der Kammeyer maßgeblich beteiligt war, stand nicht mehr das gesamte, in den 1920er Jahren genutzte Gelände zur Verfü- gung. Die »Staatliche Lehr- und Versuchsanstalt für Viehhaltung Pillnitz« übernahm im Jahr 1949 den südwestlichen Teil des ehemaligen Lehrgartens für den Aufbau eines neuen großen Lehrbienenstandes (Bienengarten). Die verbleibende Fläche gestaltete die Versuchs- und Forschungsanstalt ab 1950 als Schaugarten aus, nachdem den letzten Kleingartenpächtern gekündigt werden konnte.

Der Schaugarten sollte "als Zieranlage auch Lehr- und Anschauungsgarten sein und gleichzeitig ein Aufenthaltsraum für [die] Betriebsangehörigen werden «. ${ }^{9}$

Diese Intention zeigt ein Gestaltungsplan aus dem Jahr 1950 (Abb. 4), als Planverfasser und Zeichner ist Gottfried Schramm, der stellvertretende Abteilungsleiter der Abteilung Garten- und Landschaftsgestaltung, verzeichnet. Es ist jedoch zu vermuten, dass Kammeyer maßgeblich an der Ausarbeitung beteiligt war. Die Umsetzung der Planung erfolgte zwischen 1950 und 1951. In den nachfolgenden Jahren wurden lediglich kleinere Umgestaltungen an der Anlage vorgenommen, der Garten blieb in seiner Grundstruktur weitgehend erhalten.

Der Schaugarten enthielt neben einem 
hölzernen Gartenhaus und einem Unterrichtsplatz im Freien mehrere Sondergärten, darunter einen Rosen-, einen Stein-, einen Stauden- und einen Primelgarten sowie zwei Wechselflorbeete, die im Entwurfsplan ebenfalls als Sondergärten bezeichnet werden. Nach Kammeyer war die Form der Sondergärten für den Lehrgarten besonders geeignet, da thematisch geordnet verschiedene Pflanzenarten und -sorten präsentiert werden konnten.

Zudem bot der Schaugarten aber auch, wie von Kammeyer vorgesehen, die Möglichkeit der Erholung. Nach seinen Entwürfen wurden in der institutseigenen Tischlerei hölzerne Liegepritschen, Gartenbänke und Papierkörbe gebaut und auf der zentralen Rasenfläche aufgestellt (Abb. 5). ${ }^{10}$

Im Folgenden sollen einige Sonder- und Sortimentsbeete des Schaugartens näher vorgestellt werden (vgl. Abb. 4).

\section{Steingarten}

Der östliche Bereich des Schaugartens bis an die Schlossmauer des Pillnitzer Parks konnte für die Anlage eines Steingartens genutzt werden. Kammeyer schrieb bereits 1922 in einem Artikel in »Die Gartenwelt«:

"Steingärten gehören $\mathrm{zu}$ den reizendsten Gartengebilden, die wir kennen. Das hat man schon seit langem erkannt und besondere Steingärtchen als Sonderteile des Gartens geschaffen. $\ll^{11}$

Die von der Schlossmauer sehr steil abfallende Böschung wurde durch Aufbringen von Schuttmassen ausgezogen, modelliert und mit höheren Fels- und Steinbrocken versehen. Die Erschließung erfolgte durch Tretwege, die Überwindung des Höhenunterschiedes zum Weg durch Knüppelstufen. Oberhalb des Steingartens gab es in der

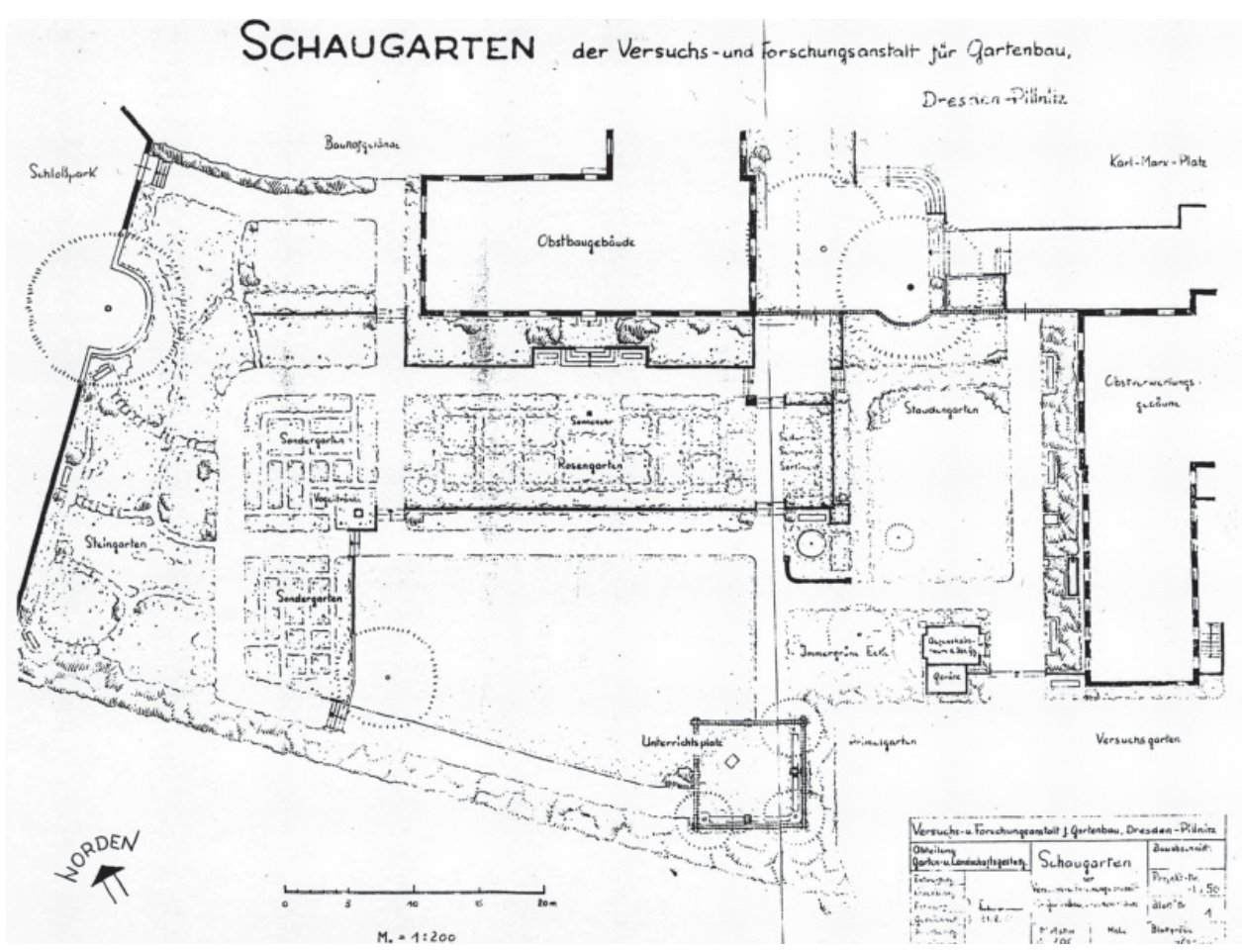

4 | Entwurf für den Schaugarten der Versuchs- und Forschungsanstalt für Gartenbau Dresden Pillnitz von 1950, Originalmaßstab 1:200 (Jahresbericht der Abteilung Garten- und Landschaftsgestaltung an der Versuchs- und Forschungsanstalt für Gartenbau Dresden-Pillnitz, Pillnitz 1951). 
Parkmauer einen Zugang zum Schlossgarten. Der Steingarten ist heute, bis auf einige Relikte durchgewachsener Wacholder-Arten, nicht mehr vorhanden.

\section{Rosenterrasse}

Die Rosenterrasse (Abb. 6), welche 1953 angelegt wurde, befand sich südlich des Schindlerbaus. Etwa 3.000 Busch- und Polyantharosen konnten in Quartierpflanzung angepflanzt werden und wurden einer Sichtung unterzogen. Dabei handelte es sich um damalige Neueinführungen sowie um bereits länger eingeführte Sorten. Die Mehrheit der Pflanzen stammte aus eigener Anzucht im Pflanzgarten. Daneben wurde ein kleiner Teil der Rosen angekauft oder mit anderen Gärten oder Betrieben getauscht. Aber auch Pflanzengeschenke, wie die Edelreiser der Firma Kordes \& Söhne aus Sparrieshoop in Holstein, trugen zur Vervollständigung der Rosensammlung bei. In den nachfolgenden Jahren wurde die Pflanzung stetig ergänzt und erweitert.

\section{Primelgarten}

Die Sammlung umfasste 50 verschiedene reine Primel- und Gartensorten. Diese wurden, aufgeteilt auf 35 Pflanzquadrate, östlich der Gärtnerunterkunft ausgepflanzt. Die Einteilung der Pflanzen erfolgte in Kissenprimeln, Glocken- und Ballprimeln sowie Dolden- und Etagenprimeln. Nachfolgend erhielten die Pflanzen eine umfassende Etikettierung, um dem Anspruch eines Sortimentsgartens für die Lehre gerecht zu werden (Abb. 7).

\section{Sonderbeete Wechselbepflanzung}

Östlich des Steingartens befanden sich beidseitig einer kleinen Treppe zwei Sonderbeete für die Wechselpflanzung mit Einjährigen. Ab 1952 wurden sie jährlich wechselnd mit verschiedenen Sortimenten bepflanzt. Die Veröffentlichung der Entwürfe fand in den Forschungsberichten der jeweiligen Jahre statt. Gestalterisch wurden viele Pflanzen einer Art beziehungsweise Sorte eingesetzt, um eine Massenwirkung zu erzielen.

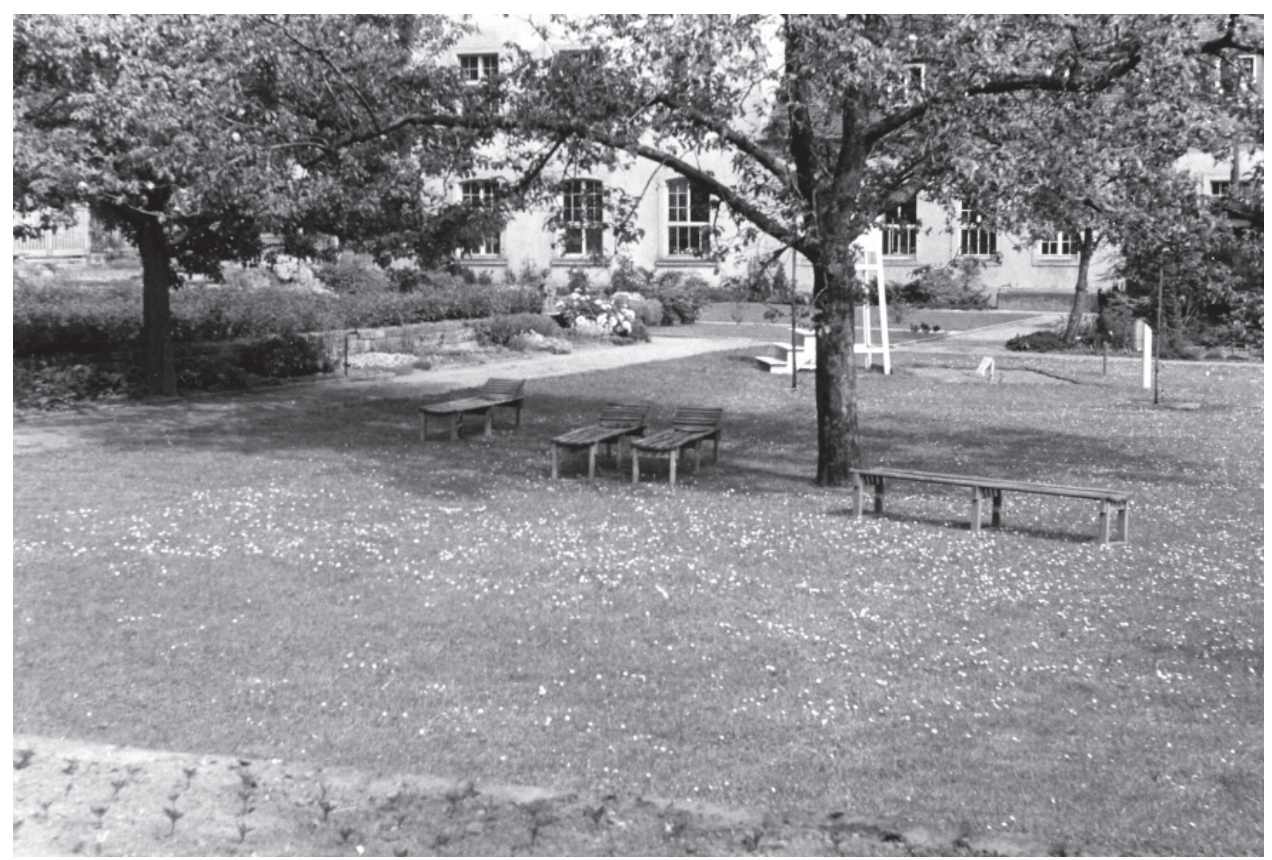

5 | Zentraler Wiesenbereich mit Liegepritschen 1957 ( Jahresbericht 1957 der Abteilung Garten- und Landschaftsgestaltung an der Versuchs- und Forschungsanstalt für Gartenbau Dresden-Pillnitz, Pillnitz 1957). 


\section{Der Kammeyergarten in Dresden-Pillnitz}

\section{Hamamelisbeet}

Das Hamamelisbeet wurde 1955 auf dem südlichen Wechselflorbeet angelegt. Vertreter aus der Familie der Hamamelidaceae verschiedene Zaubernussarten, Scheinhasel, Federbuschstrauch oder Eisenholzbaum fanden hier im Sinne eines kleinen Arboretums ihren Platz (Abb. 8). Kammeyer pflegte eine besondere Vorliebe für die Gattung der Zaubernussgewächse, worauf zahlreiche Artikel in Fachzeitschriften, eine Buchveröffentlichung zu diesem Thema sowie die wiederholte Verwendung in seinen Entwürfen hinweisen.

Kammeyer schreibt im Vorwort seines Buches »Die schönen Zaubernüsse« 1957:

"Alle diese verschiedenen Gattungen und Arten der Familie der Zaubernußgewächse sind etwas Eigenartiges und Apartes, sie zeigen viel Schönes und Besonderes, wie es vielen anderen Gehölzen nicht verliehen ist. Wer sich einmal mit ihnen beschäftigt, sie in seinem Garten angepflanzt hat und sie dort laufend beobachtet, wird nicht nur seine Freude an ihrer Schönheit haben, sondern immer wieder überrascht und begeistert sein. ${ }^{12}$

\section{Forschungsaufgaben und Pflanzen- austausch}

Mehrere Aspekte hatten unmittelbaren Einfluss auf die Pflanzenausstattung der Zierund Lehranlagen der 1950er Jahre. Die Gehölze und Stauden konnten zum Großteil in Pillnitz selbst angezogen werden. Zudem wurden Stauden- und Gehölzarten, welche in Pillnitz einer Sichtung unterzogen wurden, in den Zier- und Schauanlagen ausgepflanzt. Die Forschungstätigkeit der Lehranstalt umfasste dabei die Sichtung eingeführter Gehölz- und Staudensortimente sowie die Staudenneuheitenprüfung. Seit Beginn des 20. Jahrhunderts war eine erhebliche Zunahme von neuen Staudengattungen, -arten und -sorten zu verzeichnen. Der Prü-

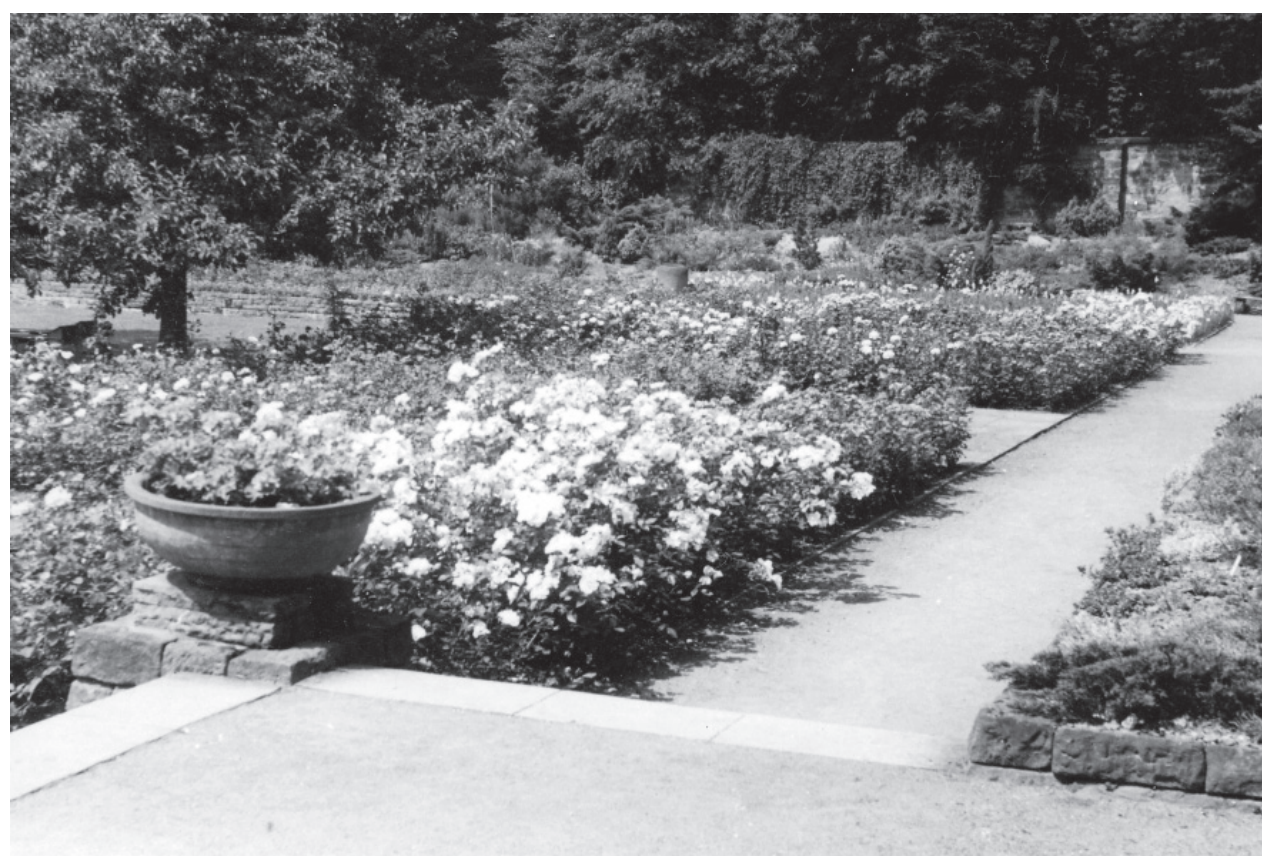

6 | Blick über die Rosenterrasse 1957 (Jahresbericht der Abteilung Garten- und Landschaftsgestaltung an der Versuchs- und Forschungsanstalt für Gartenbau Dresden-Pillnitz, Pillnitz 1957). 


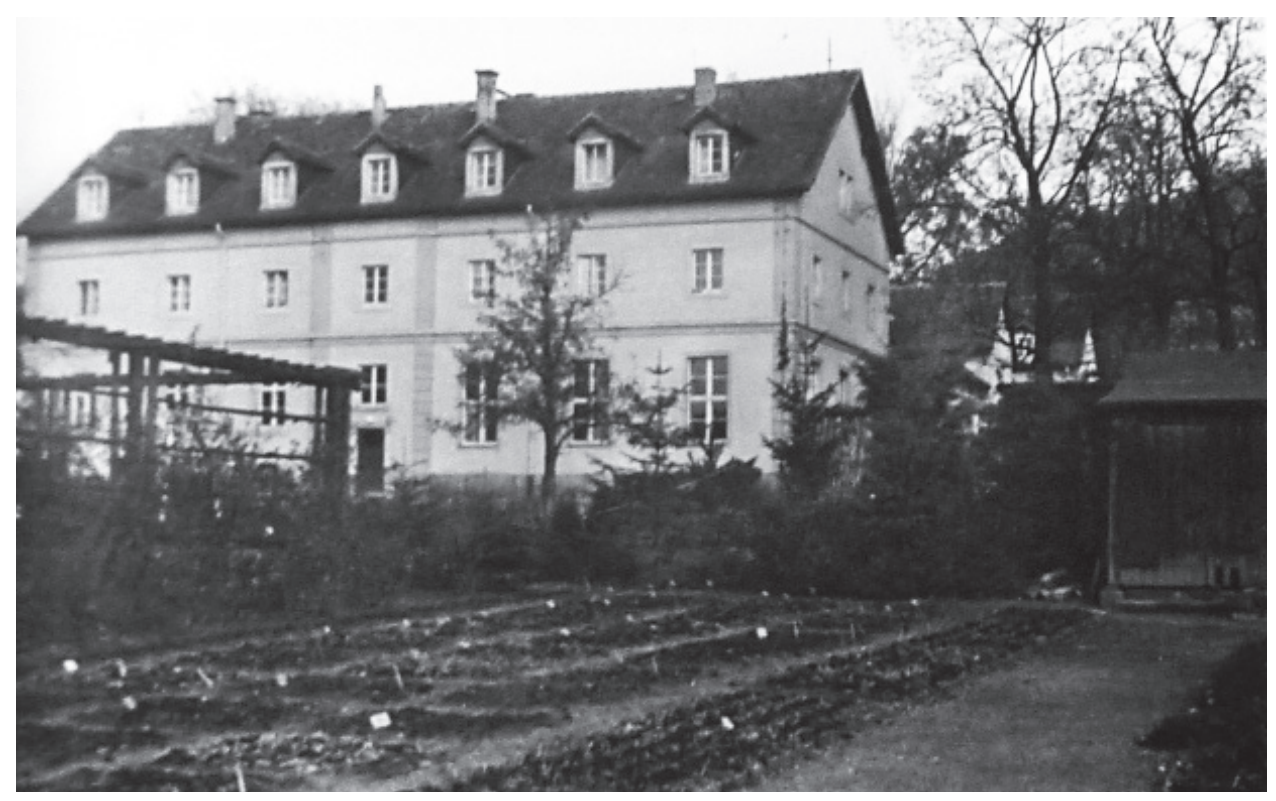

7 | Blick auf den Primelgarten 1956 (Jahresbericht der Abteilung Garten- und Landschaftsgestaltung an der Versuchs- und Forschungsanstalt für Gartenbau Dresden-Pillnitz, Pillnitz 1956).

fung des Gartenwertes dieser neuen Sorten und den daraus resultierenden Empfehlungen für ihre Verwendung kam dabei eine große Bedeutung zu. Die Forschungsanstalt in Pillnitz war eine von sieben ausgewählten Sichtungseinrichtungen im Osten Deutschlands, die mit der Staudenneuheitenprüfung betraut wurden. Verschiedene Gärtnereien sandten ihre Neuheiten ein, unter anderem auch die Staudengärtnerei Förster aus Bornim. Schwerpunkte der Staudensichtung waren dabei Iris- und Phloxsortimente.

Aber auch bereits eingeführte Stauden und Sträucher wurden in Pillnitz der Prüfung und Sichtung unterzogen. Die Forschungsanstalt konzentrierte sich dabei auf wenige Gattungen, von denen möglichst umfassende Sortimente zusammengetragen werden konnten. Laut dem Jahresbericht handelte es sich dabei um folgende Gattungen und Arten: Berg-Aster (Aster amellus), Kissen-Aster (Aster dumosus), Chrysantheme (Chrysanthemum $\mathrm{x}$ indicum), Feinstrahl (Erigeron), Primeln (Primula), Steinbrech (Saxifraga), Fetthenne (Sedum) und
Hauswurz (Sempervivum). Zu den gesichteten Gehölzgattungen gehörten Blauglockenbaum (Paulownia), zwergige Felsenbirne (Amelanchier) und Schneeball (Viburnum). ${ }^{13}$

Weiterhin konnte auch durch Austausch von Pflanzenmaterial mit anderen Institutionen, Betrieben, historischen oder botanischen Gärten das Pillnitzer Sortiment erweitert werden. In den Jahresberichten werden unter anderem die Botanischen Gärten Berlin Dahlem, Potsdam, Jena oder Tharandt genannt. Der Botanische Garten Köln schenkte der Lehr- und Forschungsanstalt ein größeres Tulpensortiment von der Bundesgartenschau in Köln. Die Anstalt erhielt auch in größeren Mengen Samen aus botanischen Gärten weltweit, so aus den Niederlanden, Jugoslawien, Peking, Polen, Österreich und Kanada. Als Austausch gab die Forschungsanstalt Vermehrungsmaterial seltener Gehölze ab.

Ebenso nutzte Kammeyer seine vielzähligen Studienreisen, um persönliche Kontakte zu Fachkollegen herzustellen. Ende August 
1951 reiste er unter anderem nach Hannover und besuchte die Herrenhäuser Gärten. In seinem dazu verfassten Bericht schrieb er:

»Ich habe viele Anregungen durch diese Reise bekommen, habe auch versucht, Beziehungen mit dortigen Fachleuten anzuknüpfen, um geeignetes Material, wie Samen und Pflanzen für die Erweiterung unserer Anlagen zu erhalten. « ${ }^{14}$

\section{Anlage des Versuchsgartens ab 1954}

Infolge der Flächenabtrennung für den Bienengarten wurde 1950 der Versuchs- und Forschungsanstalt das $1.600 \mathrm{~m}^{2}$ große Flurstück südlich des Obstverwaltungsgebäudes (heute Mitschurinbau) zur Pacht überlassen. Auf diesem Gelände war die Anlage eines Versuchsgartens angedacht, in dem Steinmaterialversuche in Form von Mauern, Treppen, und Plattenwegen exemplarisch gezeigt werden sollten. Die Präsentation weiterer Pflanzensortimente war hier vorgesehen. Kammeyer fertigte für das Gelände einen Entwurf an, der zwischen 1954 und 1957 umgesetzt wurde (Abb. 9).

Gemäß der Planung wurde eine erhöhte Terrasse mit Stützmauern aus Naturstein, im Plan als Einjahresblumen-Terrasse bezeichnet, angelegt. Die Erschließung erfolgte über mehrere, zum Teil geschwungene Treppen aus Sandstein. Vor den Aufgängen fanden Plattenbeläge aus verschiedenen sächsischen Gesteinsarten Verwendung.

Die Mauern setzten sich aus Teilstücken von jeweils zwei Metern Länge zusammen, die aus verschiedenen Gesteinsarten aus Sachsen und Thüringen bestanden. Der erhöhte Bereich wurde mit Pflanzbeeten, Gehölzen und Einjährigen ausgestaltet. An die Terrasse schloss sich ein etwa fünf Meter breiter Gang an, für die Aufstellung von Kübelpflanzen, darunter Oleander, Feige, Hortensie und Schmucklilie. Als dauerhafte Einfassung der Fläche östlich des Senkgartens setzte man Rhododendron.

\section{Familie der Hamamelidaceae}

\section{Aufpflanzung im Schaugarten Nr. $14 \mathrm{~b}$}

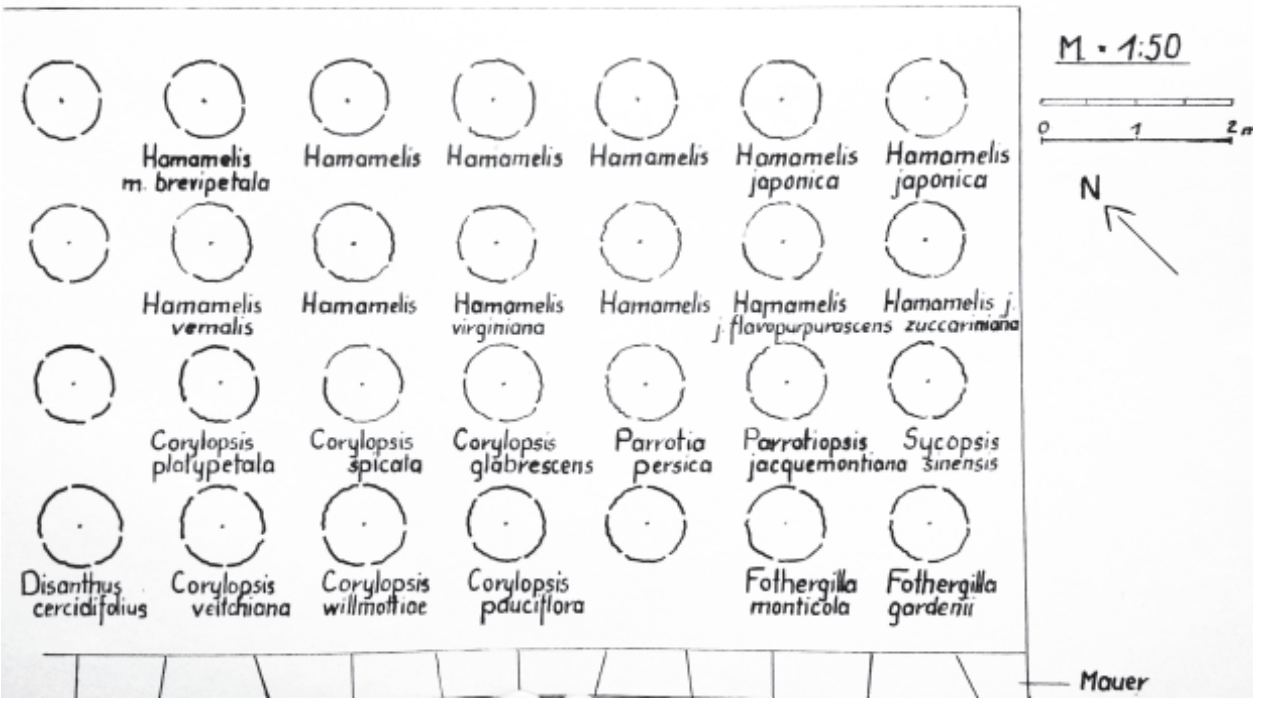

8 | Ausgeführtes Pflanzschema Hamamelisbeet 1955 (Jahresbericht der Abteilung Garten- und Landschaftsgestaltung an der Versuchs- und Forschungsanstalt für Gartenbau Dresden-Pillnitz, Pillnitz 1955). 
Im so genannten Senkgarten wurde ein rechteckiges Wasserbecken eingeordnet, das im Sommer auch zum Baden genutzt wurde. Zudem fand ein kleines, in sieben Kammern unterteiltes Wasserbecken Aufstellung, welches der Anpflanzung verschiedener Wasserpflanzen diente.

\section{Schließung der Lehranstalt 1963}

Im August 1958 ging Kammeyer in den Ruhestand. Die von ihm geleitete Abteilung Gartengestaltung an der Lehr- und Forschungsanstalt wurde mit seinem Weggang aufgelöst. Im Ruhestand widmete sich Kammeyer bis zu seinem Tod 1973 seiner publizistischen Arbeit.

Im Jahr 1963 kam es zur Schließung der Lehreinrichtung in Pillnitz. Das Institut für Obst- und Zierpflanzenbau, dessen Haupt- augenmerk der physiologischen Forschung im Obstbau galt, verblieb am Standort und bezog den Schindler- und Mitschurinbau.

Über die weitere Entwicklung des Schauund Versuchsgartens gibt es nur wenige Informationen. Sehr wahrscheinlich wurde die Gestaltung in ihren Grundzügen erhalten und nach den geänderten Nutzungsanforderungen vereinfacht. Die Kübelterrasse und der Senkgarten mit dem Wasserbecken im Versuchsgarten sind vermutlich in dieser Zeit zurückgebaut beziehungsweise verfüllt worden.

\section{Wiederaufnahme der Lehre in den 1990er Jahren und Entwicklung bis heute}

Nach der politischen Wende kehrte der Lehrbetrieb wieder in die ehemaligen Schulgebäude am Pillnitzer Platz und an der Söb-

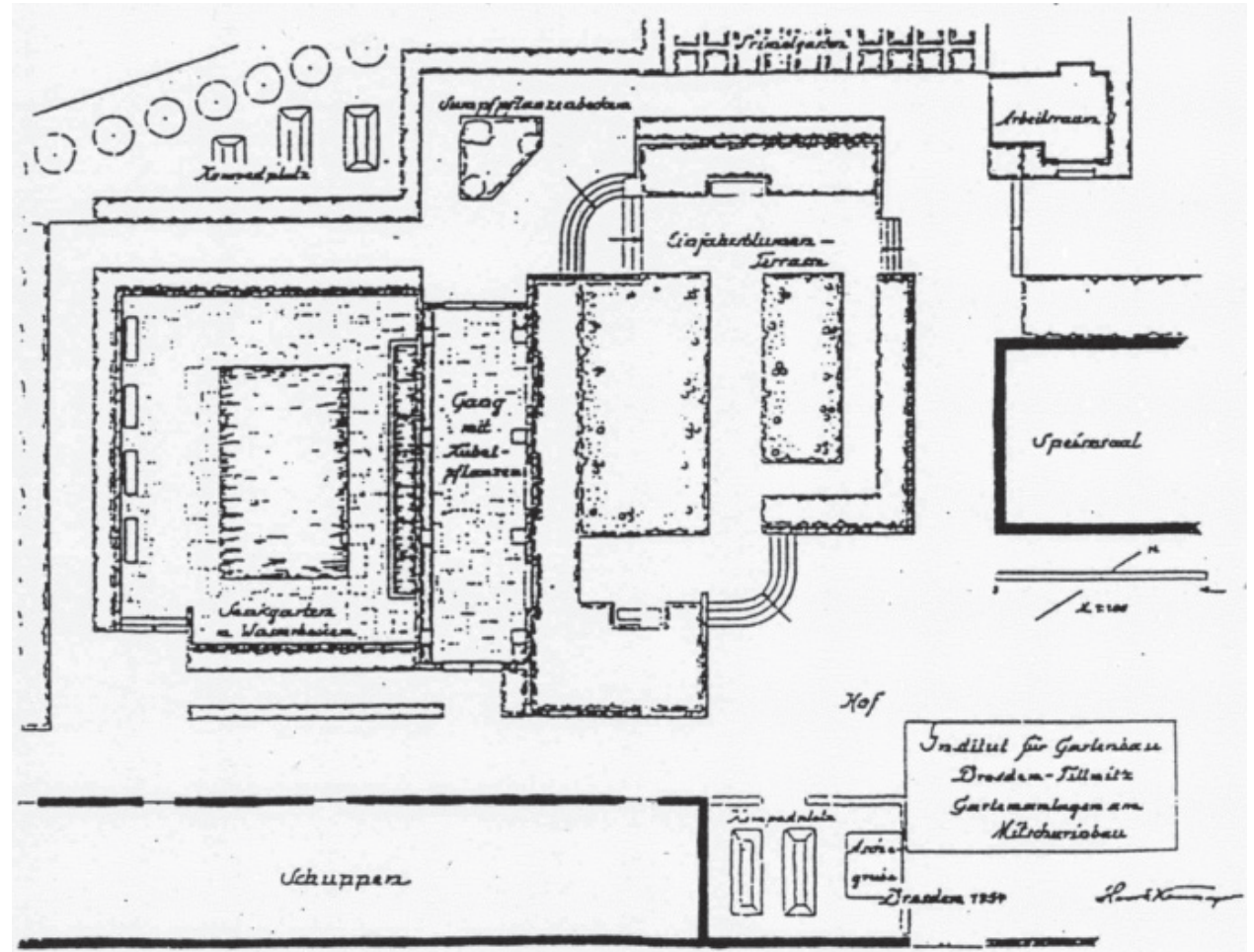

9 | Plan der »Gartenanlagen am Mitschurinbau« von 1954 (Jahresbericht der Abteilung Garten- und Landschaftsgestaltung an der Versuchs- und Forschungsanstalt für Gartenbau Dresden-Pillnitz, Pillnitz 1954). 
rigener Straße zurück. Im Jahr 1993 eröffnete die HTW den Fachbereich Pillnitz mit den Studiengängen Landespflege, Gartenbau und Landwirtschaft. Die Bereiche des ehemaligen Schau- und Versuchsgartens wurden wieder in Stand gesetzt beziehungsweise umgestaltet und der Lehrgarten wieder seiner Bestimmung zugeführt.

Ein Großteil des überkommenen Gehölzbestandes datiert aus der Zeit nach 1963 sowie nach 1990. Einige wenige Großgehölze, wie eine Farnblättrige Blutbuche (Fagus sylvatica 'Asplenifolia') oder der Urweltmammutbaum (Metasequoia glyptostroboides) stammen aus der Zeit um 1950. Ein weiteres markantes Einzelgehölz, das den Kammeyergarten durch seine Größe und Gestalt in besonderem Maße prägt, ist die KoloradoTanne (Abies concolor) auf der Wiese im ehemaligen Bienengarten. Der Baum ist bereits auf einem Luftbild von 1924 zu erkennen und somit das älteste Gehölz der Anlage.

Der jüngere Vegetationsbestand im Kammeyergarten ist - entsprechend der Funktion als Lehrgarten - sehr reich an Pflanzengattungen, -arten und -sorten. Darunter lassen sich viele botanische Besonderheiten finden, darunter Schlangenhaut-Kiefer ( $P i$ nus heldreichii), Nusseibe (Torreya nucifera),
Osagedorn (Maclura pomifera) oder verschiedene Zedernarten.

Die Gliederung der Beete im Schau- und Versuchsgarten entspricht weitgehend den Plänen von 1950 und 1954. Die thematische Struktur der Beetbepflanzung hat sich erhalten, jedoch nicht mehr an den ursprünglichen Standorten.

Der im Kammeyergarten gelegte und historisch gewachsene gestalterische und botanische Schwerpunkt soll zukünftig auch um wichtige Lehr- und Forschungsfelder der Ökosystemforschung, (Standort- und Vegetationskunde, Tierökologie, klimarelevante Prozesse des Wasserkreislaufs) erweitert werden. Prämissen sind dabei die Erhaltung und stellenweise Wiederherstellung wertvoller historischer Strukturen und Gestaltungsprinzipien. Für die Weiterentwicklung der Freianlage für Lehre und Forschung nach heutigen Erfordernissen soll die Gestaltung durch moderne Elemente, unter Berücksichtigung des historischen Kontextes, ergänzt werden. Ein wichtiger Aspekt im Sinne Kammeyers wird dabei auch die Verbesserung der Aufenthaltsqualität und Stärkung der Erholungsfunktion im Garten sein.

tenwelt, Ausgabe 36, 1922, S. 442.

1 Gröning, Gert; Wolschke-Bulmahn, Joachim: Grüne Biografien. Biografisches Handbuch zur Landschaftsarchitektur des 20. Jahrhunderts in Deutschland, Berlin/Hannover 1997, S. 174f.

2 Höhere Staatslehranstalt für Gartenbau (Hg.): Bericht 1922-25 der Höheren Staatslehranstalt für Gartenbau zu Pillnitz bei Dresden. Erstattet von dem Direktor Otto Schindler und den Abteilungsleitern, Pillnitz 1925, S. 27.

3 Staatliche Versuchs- und Forschungsanstalt für Gartenbau (Hg.): Tätigkeitsbericht der Höheren Staatslehranstalt für Gartenbau, jetzt: Staatliche Versuchs- und Forschungsanstalt für Gartenbau zu Pillnitz a. d. Elbe. Erstattet von dem Direktor Otto Schindler und den Abteilungsleitern, Pillnitz 1936, S. 49.

4 Höhere Staatslehranstalt für Gartenbau (Hg.) 1925, S. 29.

5 Ebd., S. 30.

6 Ebd.

7 Kammeyer, Hans Felix: Sondergärten, in: Die Gar-
8 Kammeyer, Hans Felix: Pillnitzer Lexikon. Hauptband nebst Abbildungsband, Dresden 1963-1965, S. 121.

9 Kammeyer, Hans Felix: Jahresbericht der Abteilung Garten- und Landschaftsgestaltung an der Versuchs- und Forschungsanstalt für Gartenbau Dresden-Pillnitz, Pillnitz 1951, S. 29.

10 Kammeyer, Hans Felix: Jahresbericht der Abteilung Gartengestaltung am Institut für Gartenbau der Deutschen Akademie der Landwirtschaftswissenschaften zu Berlin, Dresden-Pillnitz 1955, S. 34-36.

11 Kammeyer 1922, S. 444.

12 Kammeyer, Hans Felix: Die schönen Zaubernüsse, Wittenberg 1957, S. 6f .

13 Kammeyer, Hans Felix: Jahresbericht der Abteilung Gartengestaltung am Institut für Gartenbau der Deutschen Akademie der Landwirtschaftswissenschaften zu Berlin, Dresden-Pillnitz 1952, S. 40-50. 14 Kammeyer 1951, S. 14. 